\title{
Magnetic Field Simulation for Circumferential Magnetic Phase Produced in Steam Generator Tube
}

\author{
Kwon-sang Ryu ${ }^{1 *}$, Derac Son ${ }^{2}$, Duck-gun Park ${ }^{3}$, and Jae-kap Jung ${ }^{1}$ \\ ${ }^{1}$ Korea Research Institute of Standards and Science, Daejeon 305-340, Korea \\ ${ }^{2}$ Department of Physics, Hannam University, Daejeon 300-791, Korea \\ ${ }^{3}$ Korea Atomic Energy Research Institute, Daejeon 305-600, Korea
}

(Received 23 February 2011, Received in final form 27 May 2011, Accepted 29 May 2011)

\begin{abstract}
Steam generator tubes (SGTs) in nuclear power plants (NPPs) are a boundary between the primary side generating heat by nuclear fission and the secondary side generating electric power by a turbine. The water inside the SGT is high temperature and high pressure. Therefore, defects and magnetic phases (MPs) are partly produced in non-magnetic SGT by high stresses and temperatures. This causes trouble regarding the safety of SGTs but it is difficult to detect the MP using the conventional eddy current technique (ECT). In particular, a circumferential defect $(C D)$ and circumferential magnetic phase (CMP) cannot detected by ECT. Consequently, a new method is needed to detect CDs and CMPs in SGT. A new $U$-type yoke with two types of coils was designed and the reactance signal by the CMPs and CDs in the SGT material was simulated.
\end{abstract}

Keywords: magnetic field simulation, steam generator tube, circumferential magnetic phase, circumferential defect

\section{Introduction}

SGT in NPP is a heat exchanger from a primary loop generating heat by nuclear fission to a secondary one generating electric power by a turbine. The materials used for SGT are Inconel alloys, which are non-magnetic and have good corrosion-resistance. On the other hand, MPs and defects are partly generated in the SGT due to the high temperatures and stresses because the temperature and pressure of water in SGTs are very high due to nuclear fission [1]. The temperature and pressure is approximately $280^{\circ} \mathrm{C}$ and $15.5 \mathrm{MPa}$, respectively. The eddy current (EC) method is currently the most widespread inservice technique used for a pre-service inspection of the SGT in the nuclear power industry $[2,3]$. Axial direction defects in the SGT are detected by conventional ECT but the CMPs and CDs in the SGT are quite difficult to detect using this conventional technique [4].

The permeability may be described as the ease with which a material can be magnetized. For non-ferrous metals, such as copper, brass, aluminium etc., and for austenitic stainless steels, the permeability is the same as

*Corresponding author: Tel: +82-42-868-5164

Fax: +82-42-868-5018, e-mail: ryuks@kriss.re.kr that of 'free space', i.e. the relative permeability $\left(\mu_{\mathrm{r}}\right)$ is one [4]. For ferrous metals, however, the value of $\mu_{\mathrm{r}}$ may be several hundred, which has a very large signal influence on the EC response. In addition, it is not uniform and varies greatly within the SGT due to localised stresses, heating effects etc. [4]. Internal-stress caused by drawing, straightening and other processing of the SGT also creates the MPs, which can give rise to fluctuations in the permeability. Since the ferromagnetic test piece can be saturated by strong DC magnetic field, the permeability fluctuation of the magnetic test piece will be reduced. In order to eliminate such fluctuation effects during testing, a suitable device, such as a magnetized EC probe, was employed [5]. Recently, to eliminate these EC signal fluctuations, a magnetized probe with built-in permanent magnets has been used in a SGT inspection, which results in a high signal-to-noise ratio. Although this magnetized EC probe could not provide sufficient saturation for MPs with high permeability, this strong magnetic field of the magnetized EC probe amplified the EC signal as noise. The reliability of EC in a SGT inspection will be enhanced greatly by defects around the magnetic sludge and the MP produced with a defect using a magnetic sensor [6].

In this study, a new probe was designed with a $U$-type yoke and two kinds of coils, a magnetizing coil and a 
detecting coil. The reactance induced by the CMPs and $\mathrm{CDs}$ in the Inconel 600 tube was simulated using finite element method (FEM). Using the sensor developed in this study, the CMPs and CDs of a SGT could be measured at the same speed as that of the conventional EC probe.

\section{The Principle of the Designed Sensor}

The EC is described by the diffusion equation as follows [7];

$$
(1 / \mu) \nabla^{2} \vec{A}=-\vec{J}_{s}+j \omega \sigma \vec{A}+\nabla(1 / \mu) \times(\nabla \times \vec{A})
$$

where $A$ is the vector potential, $J_{\mathrm{s}}$ is the current density, $\mu$ is the magnetic permeability, $\omega$ is the angular frequency, and $\sigma$ is the conductivity. The ECT is being applied in non-ferrous materials with a relative permeability of 1 , such as Inconel alloy, because the permeability of a magnetic material severely limits the depth of penetration of the induced EC. Therefore, it is very difficult to detect the CMPs, when the permeability and conductivity change spontaneously. A new type of sensor is needed to distinguish the CMPs and CDs with its principle based on the measurement of the impedance change in the sensor composed of a $U$-type yoke wound magnetizing coil and $B$ sensing coil [6]. This sensor detects the voltage variation induced by the impedance change caused by the presence of CDs and MPs in the structure under test.

\section{Experimental}

Inconnel alloys are non-magnetic with good corrosionresistance but axial and circumferential MPs and defects are partly generated in the SGT due to the high temperatures and stress. The existence of MP in a SGT was verified from a retired SGT (Inconel 600) of Kori-1 NPP in Korea by showing a permanent magnet attached to the fragment of a SGT containing the MP [1]. The axial defects in a SGT can be detected by conventional ECT, but the CDs and CMPs are very difficult to detect. Fig. 1 shows a schematic diagram of the reference specimen for testing the new designed sensors and simulating magnetic field. The inner diameter, outer diameter, and length of the reference specimen were $16.916 \mathrm{~mm}, 19.05 \mathrm{~mm}$ and $440 \mathrm{~mm}$, respectively. The numbers 1, 2, 3 and 4 are CMPs in the outer wall, and 5 and 6 are CDs in the inner one. AISI 1810 steel was used to simulate the CMPs. The magnetic properties of its ring specimen were measured using a hysteresis remagraph (Magnet-Physik), and the conductivity was measured using a 4-point probe [8]. The maximum permeability, coercivity, residual magnetic induc-

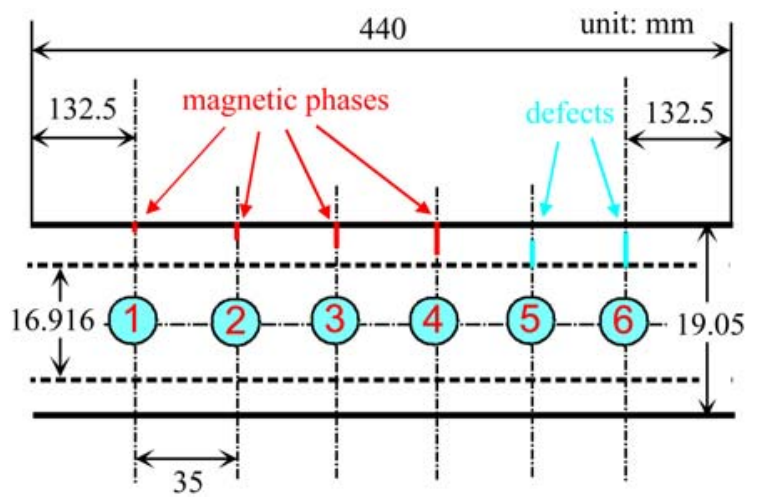

Fig. 1. Schematic diagram of the reference specimen. The numbers 1,2,3, and 4 are the CMPS in the outer wall, 5 and 6 are CDs in the inner one.

Table 1. The dimension of the CMPs and CDs in the reference specimen.

\begin{tabular}{lcccc}
\hline \hline & Sample No. & $L(\mathrm{~mm})$ & $W(\mathrm{~mm})$ & $D(\mathrm{~mm})$ \\
\hline Outer defect & 1 & 5.00 & 0.20 & $0.213(20 \%)$ \\
(Insertion of & 2 & 5.00 & 0.20 & $0.427(40 \%)$ \\
magnetic phase) & 3 & 5.00 & 0.20 & $0.639(60 \%)$ \\
& 4 & 5.00 & 0.20 & $0.852(80 \%)$ \\
\hline \multirow{2}{*}{ Inner defect } & 5 & 5.00 & 0.20 & $0.639(60 \%)$ \\
& 6 & 5.00 & 0.20 & $0.852(80 \%)$ \\
\hline
\end{tabular}

tion and conductivity of this steel were $9.80 \times 10^{-4} \mathrm{H} / \mathrm{m}$, $0.44 \mathrm{kA} / \mathrm{m}, 0.83 \mathrm{~T}$, and $6.0 \times 10^{5} \mathrm{~S} / \mathrm{m}$, respectively. Table 1 lists the dimensions and position of the CMPs and defects in the reference specimen. The CMPs and CDs had a hexahedron shape. The length and width of the CMPs and CDs were the same, and the depth was deeper in proportion to number of CMPs and CDs.

As a yoke material, $0.15 \mathrm{~mm}$ thick non-oriented silicon steel was used, and epoxy molded in vacuum. Using the thin gauged laminated core, the yoke up could be magnetized to $10 \mathrm{kHz}$. The diameter and number of wire turns used for magnetizing and $B$-sensing coils were $0.15 \mathrm{~mm}$ and 100 turns. respectively. The 10 Aturns magnetic potential with $10 \mathrm{kHz}$ magnetizing frequency was applied to the specimen using a magnetizing coil with a $U$-shape yoke.

Fig. 2 shows a schematic diagram of the sensor electronics for signal processing. The figure shows only for one sensor to simplify the diagram. A two phase lock-in amplifier was used to measure the amplitude variation and phase shift of the induced voltage due to the magnetic reluctance change caused by the presence of CMPs and CDs in the SGT because the inductance of primary winding and the phase angle between the primary current and $B$-coil voltage will be varied by the magnetic reluctance 


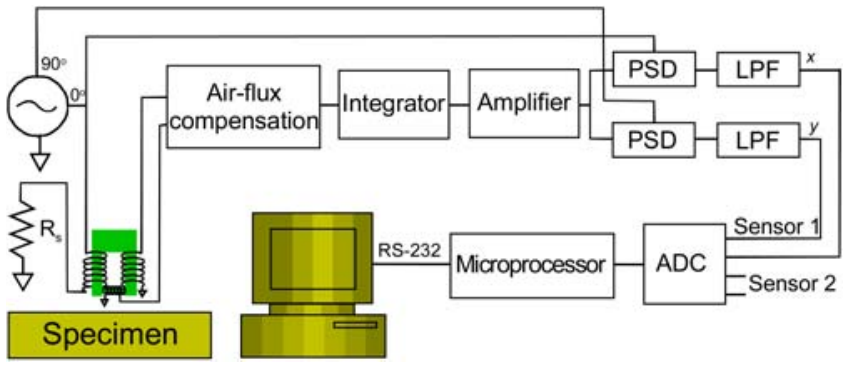

Fig. 2. Block diagram of the fabricated sensor system for measuring the CDs and CMPs.

change. When lift-off of the yoke from the surface of the test specimen was changed, the magnetic potential drop between the two pole surfaces also changed. A $H$-coil was used to reduce this lift-off effect. The induced voltage of the $H$-coil was subtracted properly from the induced voltage of the $B$-coil signal using an electronic air flux compensator. To distinguish the directions of the axial and circumferential defects and MPs, two sensors were made perpendicular to each other and were incorporated into the probe. 4-channel analog signals (2-channel analog signals per sensor with two mutually perpendicular sensors) were converted to a digital signal using a 4-channel input 16 bit Analog to Digital Converter (ADC), and the digital signals were transferred to a personal computer via a RS232C interface using 8 bit embedded microcontroller [6].

Ansoft Maxwell V12 3D software was used to calculate the reactance change caused by the presence of CMPs and CDs [9]. The $U$-type probe, which has two types of coils perpendicularly on the Inconel tube including the CMPs and CDs.

\section{Results and Discussion}

Fig. 3 shows the mesh configuration for simulating the reactance change in the CMPs and CDs. The solver for the FEM was the eddy current, the size of the mesh was

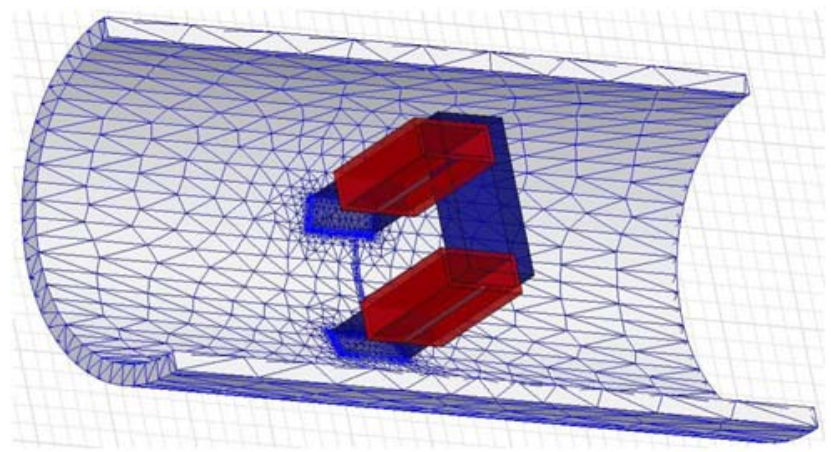

Fig. 3. Mesh configuration for simulating the reactance change.

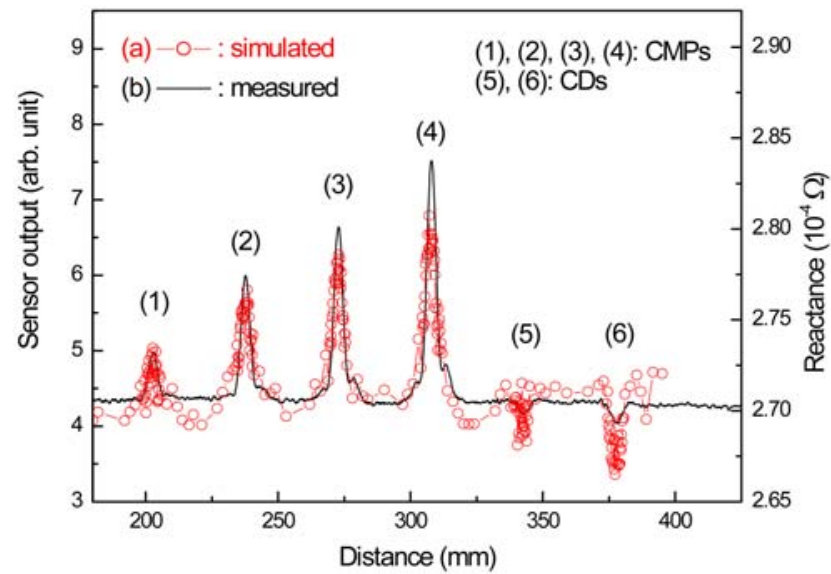

Fig. 4. (a) Simulated reactance and (b) in-phase $x$-output signal of the manufactured sensor. The numbers 1, 2, 3, and 4 are the outer CMPs, 5 and 6 are the inner CDs.

approximately 260,000 tetrahedra, and the solving frequency was $10 \mathrm{kHz}$. The magnetic potential applied to the magnetizing coil was $10 \mathrm{~A} \cdot$ turn.

Fig. 4(a) and 4(b) shows the results calculated by the FEM and the in-phase $x$-output of the sensor, respectively. As shown in Fig. 4, the reactance amplitude increased with increasing depth of the outer CMP and inner CD. The solid line, without symbols, indicates the measured reactance change of the fabricated sensor. The amplitude of the signals at the CMPs and CDs increase with depth but the phase of signals was opposite to each other. Therefore, the new sensor can measure the CMPs and CDs, and distinguish the CMPs and CDs produced in the SGT.

\section{Conclusion}

The reactance was calculated using FEM for a newly designed sensor to detect the outer CMPs and inner CDs of an Inconel tube. The reactance calculated for the CMPs and CDs in the Inconel tube agreed with the experimental results measured by the sensor. The reactance increased with the depth of the CMPs and CDs, but the phase of the signals at the CMPs and CDs are opposite each other. Therefore, the new sensor can measure the outer CMPs and inner CDs, and also distinguish the CMPs and CDs produced in the SGT. These results can be used to optimize the sensor that can detect the CMPs and CDs in the Inconel tube with speed of the conventional EC probe.

\section{References}

[1] D. G. Park, K. S. Ryu, D. Son, and Y. M. Cheong, IEEE Trans. Magn. 46, 560 (2010).

[2] P. Xiang, S. Ramakrishnam, X. Cai, P. Ramuhalli, R. Polikar, S. S. Udpa, and L. Udpa, Int. J. Appl. Electromagn. Mech. 
12, 151 (2000).

[3] S. P. Sullivan, http://www.asnt.org/publications/materialseval/ solution/may00solution/may00sol.htm.

[4] J. M. Buckley, http://joe.buckley.net/papers/eddyc.pdf.

[5] V. S. Cecco, Eddy Current Mannal, Chalk River National Laboratories, Ontario (1983).

[6] D. Son, W. I. Joung, D. G. Park, and K. S. Ryu, J. Magnet- ics 14, 97 (2009).

[7] K. S. Ryu, D. Son, D. G. Park, and Y. I. Kim, J. Appl. Phys. 107, 09E704 (2009).

[8] M. D. Janezic, DC Conductivity Measurements of Metals, NIST Tech. Note 1531 (2004).

[9] Ansoft corporation, Maxwell v13, http://www.ansoft.com. 